\title{
PARTIAL FLAG MANIFOLDS OVER A SEMIFIELD
}

\author{
G. LUSZTIG
}

Abstract. For any semifield $K$ we define a $K$-form of a partial flag manifold of a semisimple group of simply laced type over the complex numbers.

\section{INTRODUCTION}

0.1. Let $G$ be the group of simply connected-type associated in $\mathrm{MT}$, Ma, Ti], PK], to a not necessarily positive definite symmetric Cartan matrix and to the field C. We assume that a pinning of $G$ is given. It consists of a "Borel subgroup" $B^{+}$, a "maximal torus" $T \subset B^{+}$and one parameter subgroups $x_{i}: \mathbf{C} \rightarrow G, y_{i}: \mathbf{C} \rightarrow$ $G \quad(i \in I)$ analogous to those in Lus94. We have $x_{i}(\mathbf{C}) \subset B^{+}$. We fix a subset $J \subset I$. Let $\Pi^{J}$ be the subgroup of $G$ generated by $B^{+}$and by $\bigcup_{i \in J} y_{i}(\mathbf{C})$. Let $\mathcal{P}^{J}$ be the set of subgroups of $G$ which are $G$-conjugate to $\Pi^{J}$ (a partial flag manifold). As in [Lus94, 2.20] we consider the submonoid $G_{\geq 0}$ of $G$ generated by $x_{i}(a), y_{i}(a)$ with $i \in I, a \in \mathbf{R}_{\geq 0}$ and by the "vector part" $T_{>0}$ of $T$. ( $T$ is a product of $T_{>0}$ and a compact torus.) Let $K$ be a semifield. Let $\mathfrak{G}(K)$ be the monoid associated to $G, K$ by generators and relations in [L18, 3.1(i)-(viii)]. When $K=\mathbf{R}_{>0}$ this can be identified with $G_{\geq 0}$ by an argument given in [L19a].

The main result of this paper is a definition of an analogue $\mathcal{P}^{J}(K)$ of the partial flag manifold $\mathcal{P}^{J}$ in the case where $\mathbf{C}$ is replaced by any semifield $K$. This is a set $\mathcal{P}^{J}(K)$ with an action of the monoid $\mathfrak{G}(K)$.

A part of our argument involves a construction of an analogue of the highest weight integrable representations of $G$ when $G$ is replaced by the monoid $\mathfrak{G}(K)$. The possibility of such a construction comes from the positivity properties of the canonical basis Lus93. A key role in our argument is played by a classical theorem of Kostant which describes any flag manifold by a system of quadratic equations.

0.2. In this subsection we assume that our Cartan matrix is of finite-type. If $K=$ $\mathbf{R}_{>0}$, the set $\mathcal{P}^{J}(K)$ coincides with the subset $\mathcal{P}_{\geq 0}^{J}$ of $\mathcal{P}^{J}$ defined in Lus98. If $K$ is the semifield $\mathbf{Z}$ and $J=\emptyset$, a definition of the flag manifold over $\mathbf{Z}$ was given in [L19b]; we expect that it agrees with the definition in this paper, but we have not proved that. In the case where $G=S L_{n}$, a form over $\mathbf{Z}$ of a Grassmannian was defined earlier in $\mathrm{SW}$.

\section{The SET $\mathcal{P}^{J}(K)$}

1.1. Let $\mathcal{X}=\operatorname{Hom}\left(T, \mathbf{C}^{*}\right)$. This is a free abelian group with basis $\left\{\omega_{i} ; i \in I\right\}$ consisting of fundamental weights. Let $\mathcal{X}^{+}=\sum_{i \in I} \mathbf{N} \omega_{i} \subset \mathcal{X}$ be the set of dominant weights. For $\lambda \in \mathcal{X}$ let $\operatorname{supp}(\lambda)$ be the set of all $i \in I$ such that $\omega_{i}$ appears with $\neq 0$

Received by the editors February 21, 2020, and, in revised form, June 24, 2020.

2010 Mathematics Subject Classification. Primary 20 G99.

The author was supported by NSF grant DMS-1855773. 
coefficient in $\lambda$. Let $\mathcal{X}_{J}^{+}=\left\{\lambda \in \mathcal{X}^{+} ; \operatorname{supp}(\lambda)=I-J\right\}, \mathcal{X}_{\bar{J}}^{+}=\left\{\lambda \in \mathcal{X}^{+} ; \operatorname{supp}(\lambda) \subset\right.$ $I-J\}$.

The irreducible highest weight integrable representations of $G$ are indexed by their highest weight, an element of $\mathcal{X}^{+}$. For $\lambda \in \mathcal{X}^{+}$let ${ }^{\lambda} V$ be a $\mathbf{C}$-vector space which is an irreducible highest weight integrable representation of $G$ indexed by $\lambda$. Let ${ }^{\lambda} P$ be the set of lines in ${ }^{\lambda} V$. Let ${ }^{\lambda} \xi^{+}$be a highest weight vector of ${ }^{\lambda} V$. Let ${ }^{\lambda} \beta$ be the canonical basis of ${ }^{\lambda} V$ (see [Lus93, 11.10]) containing ${ }^{\lambda} \xi^{+}$.

For a nonzero vector $\xi$ in a vector space $V$ we denote by $[\xi]$ the line in $V$ that contains $\xi$. Note that $\Pi^{J}$ (see 0.1 ) is the stabilizer of $\left[{ }^{\lambda} \xi\right]$ in $G$ where $\lambda \in \mathcal{X}_{J}^{+}$.

For $\lambda, \lambda^{\prime}$ in $\mathcal{X}^{+}$we define a linear map

$$
E:{ }^{\lambda} V \times{ }^{\lambda^{\prime}} V \rightarrow{ }^{\lambda} V \otimes{ }^{\lambda^{\prime}} V
$$

by $\left(\xi, \xi^{\prime}\right) \mapsto \xi \otimes \xi^{\prime}$ and a linear map

$$
\Gamma:{ }^{\lambda+\lambda^{\prime}} V \rightarrow{ }^{\lambda} V \otimes{ }^{\lambda^{\prime}} V
$$

which is compatible with the $G$-actions and takes ${ }^{\lambda+\lambda^{\prime}} \xi^{+}$to ${ }^{\lambda} \xi^{+} \otimes{ }^{\lambda^{\prime}} \xi^{+}$. Let ${ }^{\lambda, \lambda^{\prime}} P$ be the set of lines in ${ }^{\lambda} \xi^{+} \otimes{ }^{\lambda^{\prime}} \xi^{+}$. Now $E$ induces a map $\bar{E}:{ }^{\lambda} P \times{ }^{\lambda^{\prime}} P \rightarrow{ }^{\lambda, \lambda^{\prime}} P$ and $\Gamma$ induces a map $\bar{\Gamma}:{ }^{\lambda+\lambda^{\prime}} P \rightarrow{ }^{\lambda, \lambda^{\prime}} P$.

Let $\mathcal{C}$ be the set of all collections $\left\{x_{\lambda} \in{ }^{\lambda} V ; \lambda \in \mathcal{X}_{\bar{J}}^{+}\right\}$such that for any $\lambda, \lambda^{\prime}$ in $\mathcal{X}_{\bar{J}}^{+}$we have $\Gamma\left(x_{\lambda+\lambda^{\prime}}\right)=E\left(x_{\lambda}, x_{\lambda^{\prime}}\right)$. Let $\mathcal{C}^{*}$ be the set of all $\left(x_{\lambda}\right) \in \mathcal{C}$ such that $x_{\lambda} \neq 0$ for any $\lambda \in \mathcal{X}_{\bar{J}}^{+}$. Let $H$ be the group consisting of all collections $\left\{z_{\lambda} \in \mathbf{C}^{*} ; \lambda \in \mathcal{X}_{\bar{J}}^{+}\right\}$such that for any $\lambda, \lambda^{\prime}$ in $\mathcal{X}_{\bar{J}}^{+}$we have $z_{\lambda+\lambda^{\prime}}=z_{\lambda} z_{\lambda^{\prime}}$. Now $H$ acts on $\mathcal{C}$ by $\left(z_{\lambda}\right),\left(x_{\lambda}\right) \mapsto\left(z_{\lambda} x_{\lambda}\right)$. This restricts to a free action of $H$ on $\mathcal{C}^{*}$. Let ${ }^{\prime} \mathcal{P}^{J}$ be the set of orbits for this action. Note that $G$ acts on $\mathcal{C}$ by $g\left(x_{\lambda}\right)=\left(g\left(x_{\lambda}\right)\right)$. This induces a $G$-action on $\mathcal{C}^{*}$ and on ${ }^{\prime} \mathcal{P}^{J}$. We define a map $\theta: \mathcal{P}^{J} \rightarrow{ }^{\prime} \mathcal{P}^{J}$ by $g \Pi^{J} g^{-1} \mapsto H$-orbit of $\left(g\left({ }^{\lambda} \xi\right)\right)$ where $g \in G$. This is well defined since $\left({ }^{\lambda} \xi\right) \in \mathcal{C}$ and since for $g \in \Pi^{J},\left(g\left({ }^{\lambda} \xi\right)\right)$ is in the same $H$-orbit as $\left({ }^{\lambda} \xi\right)$. We show the following.

Lemma 1.2. $\theta: \mathcal{P}^{J} \rightarrow{ }^{\prime} \mathcal{P}^{J}$ is a bijection.

For $\lambda \in \mathcal{X}_{\bar{J}}^{+}$we denote by $\Pi(\lambda)$ the stabilizer of $\left[{ }^{\lambda} \xi\right]$ in $G$. Now $\theta$ is injective since if $\lambda \in \mathcal{X}_{J}^{+}$, a subgroup $\Pi \in \mathcal{P}^{J}$ is uniquely determined by the $\Pi$-stable line in ${ }^{\lambda} V$. Now let $\left(x_{\lambda}\right) \in \mathcal{C}^{*}$. We show that the $H$-orbit of $\left(x_{\lambda}\right)$ is in $\theta\left(\mathcal{P}^{J}\right)$. Let $\lambda \in \mathcal{X}_{\bar{J}}^{+}$. We have $\Gamma\left(x_{2 \lambda}\right)=E\left(x_{\lambda}, x_{\lambda}\right)$. Thus, $E_{x_{\lambda}, x_{\lambda}}$ is contained in the irreducible summand of ${ }^{\lambda} V \otimes{ }^{\lambda} V$ which is isomorphic to ${ }^{2 \lambda} V$, hence by a theorem of Kostant (see Gar82 for the finite-type case and PK for the general case), we must have $\left[x_{\lambda}\right]=g_{\lambda}\left[{ }^{\lambda} \xi\right]$ for some $g_{\lambda} \in G$. Since $\left(x_{\lambda}\right) \in \mathcal{C}^{*}$, for $\lambda, \lambda^{\prime}$ in $\mathcal{X}_{\bar{J}}^{+}$we have

$$
\bar{E}\left(\left[g_{\lambda+\lambda^{\prime}}\left({ }^{\lambda} \xi\right)\right],\left[g_{\lambda+\lambda^{\prime}}\left({ }^{\lambda^{\prime}} \xi\right)\right]\right)=\bar{\Gamma}\left(\left[g_{\lambda+\lambda^{\prime}}\left({ }^{\lambda+\lambda^{\prime}} \xi\right)\right]\right)=\bar{E}\left(\left[g_{\lambda}\left({ }^{\lambda} \xi\right)\right],\left[g_{\lambda^{\prime}}\left({ }^{\lambda^{\prime}} \xi\right)\right]\right) .
$$

Since $\bar{E}$ is injective, it follows that

$$
\left[g_{\lambda+\lambda^{\prime}}\left({ }^{\lambda} \xi\right)\right]=\left[g_{\lambda}\left({ }^{\lambda} \xi\right)\right],\left[g_{\lambda+\lambda^{\prime}}\left({ }^{\lambda^{\prime}} \xi\right)\right]=\left[g_{\lambda^{\prime}}\left({ }^{\lambda^{\prime}} \xi\right)\right],
$$

so that

(a) $g_{\lambda}^{-1} g_{\lambda+\lambda^{\prime}} \in \Pi(\lambda)$.

Assuming that $\lambda, \lambda^{\prime} \in \mathcal{X}_{J}^{+}$, we see that $g_{\lambda}^{-1} g_{\lambda+\lambda^{\prime}} \in \Pi^{J}$ and similarly $g_{\lambda^{\prime}}^{-1} g_{\lambda+\lambda^{\prime}} \in$ $\Pi^{J}$, so that $g_{\lambda^{\prime}}^{-1} g_{\lambda} \in \Pi^{J}$. Thus, there exists $g \in G$ such that for any $\lambda \in \mathcal{X}_{J}^{+}$we have $g_{\lambda}=g p_{\lambda}$ with $p_{\lambda} \in \Pi^{J}$. Replacing $g_{\lambda}$ by $g_{\lambda} p_{\lambda}^{-1}$, we see that we can assume that

(b) $g_{\lambda}=g$ for any $\lambda \in \mathcal{X}_{J}^{+}$. 
If $\lambda \in \mathcal{X}_{\bar{J}}^{+}, \lambda^{\prime} \in \mathcal{X}_{J}^{+}$, we have $\lambda+\lambda^{\prime} \in \mathcal{X}_{J}^{+}$hence by (b), $g_{\lambda+\lambda^{\prime}}=g$, so that (a) implies $g_{\lambda}^{-1} g \in \Pi(\lambda)$ and $\left[x_{\lambda}\right]=\left[g_{\lambda}\left({ }^{\lambda} \xi\right)\right]=\left[g\left({ }^{\lambda} \xi\right)\right]$. Thus for any $\lambda \in \mathcal{X}_{\bar{J}}^{+}$we have $x_{\lambda}=z_{\lambda} g\left({ }^{\lambda} \xi\right)$ for some $z_{\lambda} \in \mathbf{C}^{*}$. Since $\left(x_{\lambda}\right) \in \mathcal{C}^{*}$ and $\left(g\left({ }^{\lambda} \xi\right)\right) \in \mathcal{C}^{*}$, we necessarily have $\left(z_{\lambda}\right) \in H$. Thus the $H$-orbit of $\left(x_{\lambda}\right)$ is in the image of $\theta$. The lemma is proved.

1.3. Let $\mathcal{D}$ be the category whose objects are pairs $(V, \beta)$ where $V$ is a $\mathbf{C}$-vector space and $\beta$ is a basis of $V$; a morphism from $(V, \beta)$ to $\left(V^{\prime}, \beta^{\prime}\right)$ is a $\mathbf{C}$-linear map $f: V \rightarrow V^{\prime}$ such that for any $b \in \beta$ we have $f(b)=\sum_{b^{\prime} \in \beta^{\prime}} c_{b, b^{\prime}} b^{\prime}$ where $c_{b, b^{\prime}} \in \mathbf{N}$ for all $b, b^{\prime}$ and $c_{b, b^{\prime}}=0$ for all but finitely many $b^{\prime}$.

Let $K$ be a semifield. As in L19b we define $K^{!}=K \sqcup\{0\}$ where $\circ$ is a symbol. We extend the sum and product on $K$ to a sum and product on $K^{!}$by defining $\circ+a=a, a+\circ=a, \circ \times a=\circ, a \times \circ=\circ$ for $a \in K$ and $\circ+\circ=\circ, \circ \times \circ=\circ$. Thus $K^{!}$ becomes a monoid under addition and a monoid under multiplication. Moreover, the distributivity law holds in $K^{!}$.

A $K$-semivector space is an abelian (additive) semigroup $\mathcal{V}$ with neutral element 으 in which a map $K^{!} \times \mathcal{V} \rightarrow \mathcal{V},(k, v) \mapsto k v$ ("scalar multiplication") is given such that $\left(k k^{\prime}\right) v=k\left(k^{\prime}(v)\right),\left(k+k^{\prime}\right) v=k v+k^{\prime} v$ for $k, k^{\prime}$ in $K^{!}, v \in \mathcal{V}$ and $k\left(v+v^{\prime}\right)=k v+k v^{\prime}$ for $k \in K^{!}, v, v^{\prime}$ in $\mathcal{V}$; moreover, we assume that $k \underline{\circ}=0$ for $k \in K^{!}$.

Let $\mathcal{D}(K)$ be the category whose objects are $K$-semivector spaces $\mathcal{V}$; a morphism from $\mathcal{V}$ to $\mathcal{V}^{\prime}$ is a map $f: \mathcal{V} \rightarrow \mathcal{V}^{\prime}$ of semigroups preserving the neutral elements and commuting with scalar multiplication. For any $\mathcal{V} \in \mathcal{D}(K)$ let $\operatorname{End}(\mathcal{V})=\operatorname{Hom}_{\mathcal{D}(K)}(\mathcal{V}, \mathcal{V})$; this is a monoid under composition of maps.

For $(V, \beta) \in \mathcal{D}$ let $V(K)$ be the set of formal sums $\xi=\sum_{b \in \beta} \xi_{b} b$ with $\xi_{b} \in K^{!}$ for all $b \in \beta$ and $\xi_{b}=\circ$ for all but finitely many $b$. We can define addition on $V(K)$ by $\left(\sum_{b \in \beta} \xi_{b} b\right)+\left(\sum_{b \in \beta} \xi_{b}^{\prime} b\right)=\sum_{b \in \beta}\left(\xi_{b}+\xi_{b}^{\prime}\right) b$. We can define scalar multiplication by elements in $K^{!}$by $k\left(\sum_{b \in \beta} \xi_{b}\right)=\sum_{b \in \beta}\left(k \xi_{b}\right) b$. Then $V(K)$ becomes an object of $\mathcal{D}(K)$. The neutral element for addition is $\underline{\circ}=\sum_{b \in \beta} \circ b$. Let $f$ be a morphism from $(V, \beta)$ to $\left(V^{\prime}, \beta^{\prime}\right)$ in $\mathcal{D}$. For $b \in \beta$ we have $f(b)=\sum_{b^{\prime} \in \beta^{\prime}} c_{b, b^{\prime}} b^{\prime}$ where $c_{b, b^{\prime}} \in \mathbf{N}$. We define a map $f(K): V(K) \rightarrow V^{\prime}(K)$ by $f(K)\left(\sum_{b \in \beta} \xi_{b} b\right)=\sum_{b^{\prime} \in \beta^{\prime}}\left(\sum_{b \in \beta} c_{b, b^{\prime}} \xi_{b}\right) b^{\prime}$. Here for $c \in \mathbf{N}, k \in K^{!}$we set $c k=k+k+\cdots+k$ (c terms) if $c>0$ and $c k=0$ if $c=0$. Note that $f(K)$ is a morphism in $\mathcal{D}(K)$. We have thus defined a functor $(V, \beta) \mapsto V(K)$ from $\mathcal{D}$ to $\mathcal{D}(K)$.

Let $\lambda \in \mathcal{X}^{+}$. We have $\left({ }^{\lambda} V,{ }^{\lambda} \beta\right) \in \mathcal{D}$ hence ${ }^{\lambda} V(K) \in \mathcal{D}(K)$ is defined. For $i \in$ $I, m \in \mathbf{Z}$, the linear maps $e_{i}^{(n)}, f_{i}^{(n)}$ from ${ }^{\lambda} V$ to ${ }^{\lambda} V$ (as in L19b, 1.4]) are morphisms in $\mathcal{D}$ (we use the positivity property [Lus93, 22.1.7] of ${ }^{\lambda} \beta$; in [Lus93 this property is stated assuming that the Cartan matrix is of simply laced-type, but the same proof applies in our case). Hence they define morphisms $e_{i}^{(n)}(K), f_{i}^{(n)}(K)$ from ${ }^{\lambda} V(K)$ to ${ }^{\lambda} V(K)$. For $i \in I, k \in K$ we define $i^{k} \in \operatorname{End}\left({ }^{\lambda} V(K)\right),(-i)^{k} \in \operatorname{End}\left({ }^{\lambda} V(K)\right)$ by

$$
i^{k}(b)=\sum_{n \in \mathbf{N}} k^{n} e_{i}^{(n)}(K) b, \quad(-i)^{k}(b)=\sum_{n \in \mathbf{N}} k^{n} f_{i}^{(n)}(K) b
$$

for any $b \in{ }^{\lambda} \beta$.

For any $i \in I$ there is a well defined function $l_{i}:{ }^{\lambda} \beta \rightarrow \mathbf{Z}$ such that for $b \in{ }^{\lambda} \beta$, $t \in \mathbf{C}^{*}$ we have $i(t) b=t^{l_{i}(b)} b$. (Here $i$ is viewed as a simple coroot homomorphism $\mathbf{C} \rightarrow T$.) For $i \in I, k \in K$ we define $\underline{i}^{k} \in \operatorname{End}\left({ }^{\lambda} V(K)\right)$ by $\underline{i}^{k}(b)=k^{l_{i}(b)} b$ for any $b \in{ }^{\lambda} \beta$. As in [L19b, 1.5], the elements $i^{k},(-i)^{k}, \underline{i}^{k}$ (with $i \in I, k \in K$ ) in 
$\operatorname{End}\left({ }^{\lambda} V(K)\right)$ satisfy the relations in [L19a, 2.10(i)-(vii)] defining the monoid $\mathfrak{G}(K)$ hence they define a monoid homomorphism $\mathfrak{G}(K) \rightarrow \operatorname{End}\left({ }^{\lambda} V(K)\right)$. It follows that $\mathfrak{G}(K)$ acts on ${ }^{\lambda} V(K)$.

1.4. In the setup of 1.4 for $\lambda, \lambda^{\prime}$ in $\mathcal{X}^{+}$we can view ${ }^{\lambda} V \otimes{ }^{\lambda^{\prime}} V$ with its basis $\mathcal{S}=$ ${ }^{\lambda} \beta \otimes{ }^{\lambda^{\prime}} \beta$ as an object of $\mathcal{D}$. Hence $\left({ }^{\lambda} V \otimes{ }^{\lambda^{\prime}} V\right)(K) \in \mathcal{D}(K)$ is defined. We define $E(K):{ }^{\lambda} V(K) \times{ }^{\lambda^{\prime}} V(K) \rightarrow\left({ }^{\lambda} V \otimes{ }^{\lambda^{\prime}} V\right)(K)$ by

$$
\left(\sum_{b \in \in^{\lambda} \beta} \xi_{b} b\right),\left(\sum_{b^{\prime} \in \lambda^{\prime} \beta} \xi_{b^{\prime}}^{\prime} b^{\prime}\right) \mapsto \sum_{\left(b, b^{\prime}\right) \in \mathcal{S}} \xi_{b} \xi_{b^{\prime}}^{\prime}\left(b \otimes b^{\prime}\right) .
$$

(This is not a morphism in $\mathcal{D}(K)$.) We define a map

$$
\operatorname{End}\left({ }^{\lambda} V(K)\right) \times \operatorname{End}\left({ }^{\lambda^{\prime}} V(K)\right) \rightarrow \operatorname{End}\left(\left({ }^{\lambda} V \otimes{ }^{\lambda^{\prime}} V\right)(K)\right)
$$

by $\left.\left(\tau, \tau^{\prime}\right) \mapsto\left[b \otimes b^{\prime}\right) \mapsto E(K)\left(\tau(b), \tau^{\prime}\left(b^{\prime}\right)\right)\right]$. Composing this map with the map

$$
\mathfrak{G}(K) \rightarrow \operatorname{End}\left({ }^{\lambda} V(K)\right) \times \operatorname{End}\left({ }^{\lambda^{\prime}} V(K)\right)
$$

whose components are the maps

$$
\mathfrak{G}(K) \rightarrow \operatorname{End}\left({ }^{\lambda} V(K)\right), \quad \mathfrak{G}(K) \rightarrow \operatorname{End}\left({ }^{\lambda^{\prime}} V(K)\right)
$$

in 1.4 we obtain a map $\mathfrak{G}(K) \rightarrow \operatorname{End}\left(\left({ }^{\lambda} V \otimes{ }^{\lambda^{\prime}} V\right)(K)\right)$ which is a monoid homomorphism. Thus $\mathfrak{G}(K)$ acts on $\left({ }^{\lambda} V \otimes{ }^{\lambda^{\prime}} V\right)(K)$; it also acts on ${ }^{\lambda} V(K) \times{ }^{\lambda^{\prime}} V(K)$ (by 1.4) and the two actions are compatible with $E(K)$.

Let $\Gamma:{ }^{\lambda+\lambda^{\prime}} V \rightarrow{ }^{\lambda} V \otimes{ }^{\lambda^{\prime}} V$ be as in 1.1. For $b \in{ }^{\lambda+\lambda^{\prime}} \beta$ we have

$$
\Gamma(b)=\sum_{\left(b_{1}, b_{1}^{\prime}\right) \in \mathcal{S}} e_{b, b_{1}, b_{1}^{\prime}} b_{1} \otimes b_{1}^{\prime}
$$

where $e_{b, b_{1}, b_{1}^{\prime}} \in \mathbf{N}$. (This can be deduced from the positivity property Lus93, 14.4.13(b)] of the homomorphism $r$ in Lus93, 1.2.12].) Thus $\Gamma$ is a morphism in $\mathcal{D}$ hence $\Gamma(K):{ }^{\lambda+\lambda^{\prime}} V(K) \rightarrow\left({ }^{\lambda} V \otimes{ }^{\lambda^{\prime}} V\right)(K)$ is a well defined morphism in $\mathcal{D}(K)$. Note that $\Gamma(K)$ is compatible with the action of $\mathfrak{G}(K)$ on the two sides.

1.5. In the setup of 1.4 let $\mathcal{C}(K)$ be the set of all collections $\left\{x_{\lambda} \in{ }^{\lambda} V(K) ; \lambda \in \mathcal{X}_{\bar{J}}^{+}\right\}$ such that for any $\lambda, \lambda^{\prime}$ in $\mathcal{X}_{\bar{J}}^{+}$we have $\Gamma(K)\left(x_{\lambda+\lambda^{\prime}}\right)=E(K)\left(x_{\lambda}, x_{\lambda^{\prime}}\right)$. Let $\mathcal{C}^{*}(K)$ be the set of all $\left(x_{\lambda}\right) \in \mathcal{C}(K)$ such that $x_{\lambda} \neq \underline{\circ}$ for any $\lambda \in \mathcal{X}_{\bar{J}}^{+}$. Let $H(K)$ be the group (multiplication component by component) consisting of all collections $\left\{z_{\lambda} \in K ; \lambda \in \mathcal{X}_{\bar{J}}^{+}\right\}$such that for any $\lambda, \lambda^{\prime}$ in $\mathcal{X}_{\bar{J}}^{+}$we have $z_{\lambda+\lambda^{\prime}}=z_{\lambda} z_{\lambda^{\prime}}$. Now $H(K)$ acts on $\mathcal{C}(K)$ by $\left(z_{\lambda}\right),\left(x_{\lambda}\right) \mapsto\left(z_{\lambda} x_{\lambda}\right)$. This restricts to a free action of $H(K)$ on $\mathcal{C}^{*}(K)$. Let $\mathcal{P}^{J}(K)$ be the set of orbits for this action. Note that $\mathfrak{G}(K)$ acts on $\mathcal{C}(K)$ by acting component by component (see 1.4); we use that $E(K), \Gamma(K)$ are compatible with the $\mathfrak{G}(K)$-actions (see 1.5). This induces a $\mathfrak{G}(K)$-action on $\mathcal{P}^{J}(K)$.

1.6. In this subsection we assume that $K=\mathbf{R}_{>0}$. If $\left(x_{\lambda}\right) \in \mathcal{C}^{*}(K)$, we can view $\left(x_{\lambda}\right)$ as an element of $\mathcal{C}^{*}$ by viewing ${ }^{\lambda} V(K)$ as a subset of ${ }^{\lambda} V$ in an obvious way. The inclusion $\mathcal{C}^{*}(K) \subset \mathcal{C}^{*}$ is compatible with the actions of $H(K)$ and $H$ (we have $H(K) \subset H$ ) hence it induces an (injective) map $\mathcal{P}^{J}(K) \rightarrow{ }^{\prime} \mathcal{P}^{J}$. Composing this with the inverse of the bijection $\mathcal{P}^{J} \rightarrow{ }^{\prime} \mathcal{P}^{J}$ (see 1.3) we obtain an injective map $\mathcal{P}^{J}(K) \rightarrow \mathcal{P}^{J}$. We define $\mathcal{P}_{>0}^{J}$ to be the image of this map.

Assuming further that our Cartan matrix is of finite-type, we show that the last definition of $\mathcal{P}_{\geq 0}^{J}$ agrees with the definition in [Lus98]. Applying [Lus98, 3.4] to a 
$\lambda \in \mathcal{X}_{J}^{+}$with large enough coordinates we see that $\mathcal{P}_{\geq 0}^{J}$ (in the new definition) is contained in $\mathcal{P}_{>0}^{J}$ (in the definition of [us98]). The reverse inclusion follows from Lus98, 3.2].

1.7. Any homomorphism of semifields $K \rightarrow K^{\prime}$ induces in an obvious way a map $\mathcal{P}^{J}(K) \rightarrow \mathcal{P}^{J}\left(K^{\prime}\right)$

1.8. We expect that when $K^{\prime}$ is the semifield $\{1\}$ with one element, one can identify $\mathcal{P}^{\emptyset}\left(K^{\prime}\right)$ with the set of pairs $\left(a, a^{\prime}\right)$ in the Weyl group $W$ of $G$ such that $a \leq a^{\prime}$ for the standard partial order of $W$. If $K$ is any semifield one can also expect that the fibre of the map $\mathcal{P}^{\emptyset}(K) \rightarrow \mathcal{P}^{\emptyset}(\{1\})$ induced by the obvious map $K \rightarrow\{1\}$ (see 1.8) at the element corresponding to $\left(a, a^{\prime}\right)$ is in bijection with $K^{\left|a^{\prime}\right|-|a|}$ where $a \mapsto|a|$ is the length function on $W$.

\section{The SEMIRING $M(K)$}

2.1. In this section we assume that our Cartan matrix is of finite-type. Let $K$ be a semifield. Let $M(K)=\bigoplus_{\lambda \in \mathcal{X}_{\bar{J}}^{+}}{ }^{\lambda} V(K)$ viewed as a monoid under addition and with scalar multiplication by elements of $K^{!}$.

We define a multiplication $\mu: M(K) \times M(K) \rightarrow M(K)$ which is "bilinear" with respect to addition and scalar multiplication and satisfies $\mu\left(b_{1}, b_{1}^{\prime}\right)=\sum_{b \in \lambda+\lambda^{\prime} \beta} e_{b, b_{1}, b_{1}^{\prime}} b$ where $\lambda \in \mathcal{X}_{\bar{J}}^{+}, \lambda^{\prime} \in \mathcal{X}_{\bar{J}}^{+}, b_{1} \in{ }^{\lambda} \beta, b_{1}^{\prime} \in{ }^{\lambda^{\prime}} \beta$, and $e_{b, b_{1}, b_{1}^{\prime}} \in \mathbf{N}$ (viewed as an element of $\left.K^{!}\right)$is as in the definition of $\Gamma(K)$ in 1.5. so that it comes from the homomorphism $r$ in [Lus93, 1.2.12]. This can be viewed as a direct sum of "transposes" of maps like $\Gamma(K)$. From the properties of $r$ we see that the multiplication $\mu$ is associative and commutative; it is clearly distributive with respect to addition. This multiplication has a unit element, given by the unique element in $\beta^{\lambda}$ with $\lambda=0$. Note that $M(K)$ is a semiring. Now $M(K)$ can be viewed as a form over $K$ of the coordinate ring of $G / U^{+}$where $U^{+}$is the unipotent radical of $B^{+}$. Let $M^{\prime}(K)$ be the set of maps $M(K) \rightarrow K^{!}$which are compatible with addition, multiplication, and with scalar multiplication by elements of $K^{!}$, take the unit element of $M(K)$ to the unit element of $K^{!}$, and take the element with all components equal to $\underline{\circ}$ to $\circ \in K^{!}$. It is easy to show that $M^{\prime}(K)$ is in canonical bijection with $\mathcal{C}(K)$.

\section{REFERENCES}

[Gar82] Devra Garfinkle, A new construction of the Joseph ideal, ProQuest LLC, Ann Arbor, MI, 1982. Thesis (Ph.D.)-Massachusetts Institute of Technology. MR2941017

[Lus93] George Lusztig, Introduction to quantum groups, Progress in Mathematics, vol. 110, Birkhäuser Boston, Inc., Boston, MA, 1993. MR1227098

[Lus94] G. Lusztig, Total positivity in reductive groups, Lie theory and geometry, Progr. Math., vol. 123, Birkhäuser Boston, Boston, MA, 1994, pp. 531-568, DOI 10.1007/978-1-46120261-5_20. MR1327548

[Lus98] G. Lusztig, Total positivity in partial flag manifolds, Represent. Theory 2 (1998), 70-78, DOI 10.1090/S1088-4165-98-00046-6. MR1606402

[L19a] G. Lusztig, Total positivity in reductive groups, II, Bull. Inst. Math. Acad. Sin. (N.S.) 14 (2019), no. 4, 403-459, DOI 10.21915/bimas.2019402. MR 4054343

[L18] G. Lusztig, Total positivity in reductive groups, II, Bull. Inst. Math. Acad. Sin. (N.S.) 14 (2019), no. 4, 403-459, DOI 10.21915/bimas.2019402. MR 4054343

[L19b] G. Lusztig, The flag manifold over the semifield Z, arXiv:1912.13329

[Ma] Richard Marcuson, Tits' systems in generalized nonadjoint Chevalley groups, J. Algebra 34 (1975), 84-96, DOI 10.1016/0021-8693(75)90195-7. MR399295 
[MT] R. V. Moody and K. L. Teo, Tits' systems with crystallographic Weyl groups, J. Algebra 21 (1972), 178-190, DOI 10.1016/0021-8693(72)90016-6. MR320165

[PK] Dale H. Peterson and Victor G. Kac, Infinite flag varieties and conjugacy theorems, Proc. Nat. Acad. Sci. U.S.A. 80 (1983), no. 6, i, 1778-1782, DOI 10.1073/pnas.80.6.1778. MR 699439

[SW] David Speyer and Lauren Williams, The tropical totally positive Grassmannian, J. Algebraic Combin. 22 (2005), no. 2, 189-210, DOI 10.1007/s10801-005-2513-3. MR2164397

[L19b] Mogens Esrom Larsen, Summa summarum, CMS Treatises in Mathematics, Canadian Mathematical Society, Ottawa, ON; A K Peters, Ltd., Wellesley, MA, 2007. [Author name on title page: Morgens Esrom Larsen]. MR2346609

[Ti] J. Tits, Resumé de cours, Annuaire Collège de France 81 (1980-81), 75-87.

Department of Mathematics, Massachusetts Institute of Technology, Cambridge, MAssachusetTs 02139

Email address: gyuri@mit.edu 